Meta

Journal des traducteurs

Translators' Journal

\title{
Comment le sous-titrage et le doublage peuvent modifier la perception d'un film
}

\author{
Analyse contrastive des versions sous-titrées et doublées en \\ français du fillm d'Elia Kazan, A Streetcar Named Desire (1951)
}

\section{Nathalie Ramière}

Volume 49, numéro 1, avril 2004

Traduction audiovisuelle

Audiovisual Translation

URI : https://id.erudit.org/iderudit/009026ar

DOI : https://doi.org/10.7202/009026ar

Aller au sommaire du numéro

Éditeur(s)

Les Presses de l'Université de Montréal

ISSN

0026-0452 (imprimé)

1492-1421 (numérique)

Découvrir la revue

Citer cet article

Ramière, N. (2004). Comment le sous-titrage et le doublage peuvent modifier la perception d'un film : analyse contrastive des versions sous-titrées et doublées en français du film d'Elia Kazan, A Streetcar Named Desire (1951). Meta, 49(1), 102-114. https://doi.org/10.7202/009026ar
Résumé de l'article

Avec les longs métrages célèbres, devenus même parfois films-cultes, la question de la traduction (principalement sous la forme de versions sous-titrées ou doublées) se pose de façon d'autant plus pertinente que les critiques et les spectateurs ont souvent tendance à amalgamer film original et versions traduites. Il semble donc important de réévaluer ces traductions et leur influence sur la réception d'un film.

À partir d'une analyse contrastive détaillée de la version originale du film de Kazan, A Streetcar Named Desire (1951) et deux de ses versions (sous-titrée et doublée en français), je me propose d'exposer les répercussions des choix respectifs faits par les traducteurs sur deux aspects majeurs dans la signification esthétique et symbolique du film, à savoir la présentation des personnages et leurs relations ainsi que les questions de transferts culturels. 


\title{
Comment le sous-titrage et le doublage peuvent modifier la perception d'un film. Analyse contrastive des versions sous-titrée et doublée en français du film d'Elia Kazan, A Streetcar Named Desire (1951)'
}

\author{
NATHALIE RAMIÈRE \\ University of Alberta, Alberta, Canada \\ nathalie.ramiere@eudoramail.com
}

\begin{abstract}
RÉSUMÉ
Avec les longs métrages célèbres, devenus même parfois films-cultes, la question de la traduction (principalement sous la forme de versions sous-titrées ou doublées) se pose de façon d'autant plus pertinente que les critiques et les spectateurs ont souvent tendance à amalgamer film original et versions traduites. II semble donc important de réévaluer ces traductions et leur influence sur la réception d'un film.

À partir d'une analyse contrastive détaillée de la version originale du film de Kazan, $A$ Streetcar Named Desire (1951) et deux de ses versions (sous-titrée et doublée en français), je me propose d'exposer les répercussions des choix respectifs faits par les traducteurs sur deux aspects majeurs dans la signification esthétique et symbolique du film, à savoir la présentation des personnages et leurs relations ainsi que les questions de transferts culturels.
\end{abstract}

\begin{abstract}
In the case of 'cult-movies,' translations (mostly subtitled and dubbed versions) are often authoritative or taken for granted, to the extent that the reception of the original movie and that of the "translated" versions are generally undifferentiated by movie critics. There is, therefore, a major interest in reconsidering these translations and their impact on the question of reception.

Through a detailed contrastive analysis of the original English version of Kazan's $A$ Streetcar Named Desire (1951) with both its subtitled and dubbed French versions, this paper intends to analyze the impacts of the respective choices made by the translators on two issues deemed crucial to the aesthetic and symbolic meaning of the film, namely: the interpersonal relationships between the characters, and cultural transfers.
\end{abstract}

\section{MOTS-CLÉS/KEYWORDS}

personnages, réception, relations interpersonnelles, transferts culturels, Un tramway nommé Désir

Malgré ses enjeux considérables, la question de la traduction audiovisuelle est longtemps restée ignorée par les traductologues, probablement, comme le laisse entendre Dirk Delabastita parce qu'elle a été associée au statut médiocre encore parfois accordé aux médias et à la culture de masse (1990: 97). Cependant, comme il modifie nécessairement le «signe filmique» original, ce type particulier de traduction a une influence déterminante sur la réception d'un long métrage, d'un film publicitaire ou d'un documentaire pour la télévision dans des pays étrangers. 
Depuis quelques années cependant, la recherche dans ce domaine s'est beaucoup développée et a tenté de mieux comprendre les enjeux et les impacts des différents modes de traduction audiovisuelle sur le public. Or, dans le cas de longs métrages devenus célèbres, voire films-cultes, la question de la traduction (principalement sous la forme de versions sous-titrées ou doublées) se pose de façon d'autant plus pertinente que les critiques et les spectateurs ont souvent tendance à amalgamer le film original et sa version traduite au point de ne plus les distinguer. Comme le font remarquer José Lambert et Dirk Delabastita, «[L]es origines étrangères du discours audiovisuel sont [...] ignorées» (1996: 48). Il semble donc crucial de réévaluer les traductions de ces films célèbres et leur marque sur la réception auprès du public, d'autant plus qu'elles ont joué - et continuent de jouer un rôle artistique, culturel, social, voire idéologique, très important. L'étude de la réception est cependant très délicate puisque, à défaut de pouvoir, par exemple recueillir les réactions d'un nombre suffisant de spectateurs à la sortie d'un film, la seule source à la disposition du chercheur est la lecture des critiques qui peuvent représenter, bien évidemment, un point de vue très différent de celui du public dans son ensemble. L'étude systématique du lien entre traduction audiovisuelle et réception des films reste un domaine d'avenir.

\section{Un film-culte: A Streetcar Named Desire}

Le film d'Elia Kazan, A Streetcar Named Desire, sorti aux États-Unis en 1951, a lui même acquis ce statut de film-culte, en particulier pour avoir, à l'époque, révélé le très jeune Marlon Brando, devenu depuis un «monstre sacré» du cinéma. La version française, sortie l'année suivante sous le titre Un tramway nommé Désir, avait, comme dans d'autres pays, choqué certains esprits peu habitués aux thèmes subversifs suggérés à l'écran ou avait, au contraire, été applaudi par d'autres pour sa technique de mise en scène et d'interprétation assez révolutionnaire pour l'époque. Le film était une reprise de la pièce écrite par Tennessee Williams, que Kazan avait déjà mise en scène pour Broadway quelques années auparavant. L'immense succès de la pièce (855 représentations en deux ans [Kolin, 2000:1]) avait alors attiré l'attention de producteurs hollywoodiens, qui persuadèrent Elia Kazan de diriger le film en gardant pratiquement la même équipe d'acteurs que celle qui avait fait le succès de la pièce. Ils exigèrent seulement que Jessica Tandy soit remplacée par l'actrice anglaise Vivien Leigh dans le rôle de Blanche DuBois. Malgré les changements au script qui furent imposés par la censure (en particulier les références à l'homosexualité et au viol de Blanche par Stanley), Tennessee Williams lui-même déclara: "This is the first time I have seen a stage play actually increase with stature when transferred to the screen" (Kolin, 2000: 151).

L'histoire dépeint le contraste entre le monde réaliste et violent de Stanley (ouvrier, fils d'immigrant polonais, issu de la société urbaine du nord des États-Unis en plein essor industriel) et le monde d'illusions et de rêves déchus de Blanche DuBois, sa belle-sœur (qui représente, elle, la société décadente des planteurs du vieux Sud en train de disparaître). Les deux protagonistes, tels Éros et Thanatos, entrent peu à peu dans un jeu pervers d'attraction et de répulsion qui mènera à la destruction mentale de Blanche. Ce climat de tension repose sur un ensemble complexe de signes à la fois linguistiques, visuels (dénotés par l'éclairage particulier, les contrastes de noir et blanc, la proxémique) et acoustiques (en particulier les bruits de 
la rue et les motifs musicaux d'Alex North qui accompagnent les protagonistes principaux). Cette tension sert de cadre à Tennessee Williams pour tenter de définir le désir, en célébrant avec ambiguïté "the male form as sexual icon while boldly interrogating female desire, and rejoicing simultaneously in the seduction of both genders » (Kolin, 2000: 2). L'histoire présente des personnages subtils et complexes et s'inscrit dans un contexte fortement marqué culturellement (par son ancrage historique, géographique et socioculturel particulier) - deux éléments susceptibles de poser des défis aux traducteurs et, en même temps, si importants pour la signification esthétique et symbolique du film que leur modification éventuelle par la traduction pourrait changer totalement la réception du film. En me basant sur une approche contrastive détaillée de la version originale du film et deux de ses versions sous-titrée et doublée en français ${ }^{2}$, je me propose donc de mettre en évidence les répercussions des choix respectifs faits par le sous-titreur et le doubleur sur ces deux questions c'est-à-dire, d'une part, les questions de transferts culturels et, d'autre part, la présentation des personnages et de leurs relations.

\section{Démarche d'analyse}

Dans un premier temps, j'analyserai les questions de transferts culturels pour examiner comment la version doublée et la version sous-titrée ont tenté de suggérer le contexte socioculturel complexe du film. Cette analyse se fera dans la perspective de la pragmatique interculturelle. Puisque "[the] users belong to a different cultural world than those for whom the signs were originally intended, we can predict major clashes between these two worlds» (Niemeier, 1991: 145). Je me pencherai ensuite sur la façon dont les versions sous-titrée et doublée peuvent influer sur la présentation des personnages principaux du film et leurs relations. Je me baserai ici, en particulier, sur l'étude menée par Hatim et Mason (1997) au sujet des effets du sous-titrage sur la représentation des relations interpersonnelles dans le film français Un cour en hiver ${ }^{3}$, en cherchant à montrer si leurs conclusions peuvent être confirmées dans le cas du film d'Elia Kazan.

Bien que la polémique traditionnelle sur les avantages supposés du sous-titrage et du doublage semble être restée stérile pour le domaine, les analyses contrastives de nature empirique - encore assez rares - peuvent cependant mettre en évidence certains problèmes spécifiques soulevés par les modalités même de ces deux types de traduction audiovisuelle. Il est possible, en particulier, grâce à ces analyses constrastives, de se pencher de façon pertinente sur certaines questions de transfert culturel (question de la traduction des noms propres, des références interlinguistiques ou des références culturelles spécifiques, par exemple) ou de normes linguistiques (registre de langue, question de l'argot, des accents, des marqueurs d'hésitation, etc.). Mon but, par conséquent, n'est pas de mettre en opposition binaire sous-titrage et doublage, comme cela a été fait de nombreuses fois, ni même de critiquer les différents choix faits par les traducteurs-adapteurs (car nous devons garder à l'esprit les contraintes techniques et financières imposées à la traduction audiovisuelle, et ainsi les limites de toute analyse constrastive), mais au contraire de m'attacher à montrer que les jugements de valeur souvent élitistes portés sur le sous-titrage ou le doublage $^{4}$, doivent sérieusement être remis en question. 


\section{Les transferts culturels dans A Streetcar Named Desire}

Étant donné que le contexte socioculturel du film joue un rôle très important, la façon dont il est rendu par la traduction peut en modifier l'image chez le spectateur. Dans ce domaine, comme le souligne George Lang, les sous-titres ont tendance à évoquer constamment la présence de «l'étranger» (1996: 399) et donc à rappeler aux spectateurs qu'ils ont affaire à une traduction. Le doublage, lui, tend à effacer cette distance (même s'il n'y pas consensus sur ce point), le danger étant que le spectateur interprète «la sphère de référence culturelle» (Lang, 1996: 400) selon la perspective de sa propre culture, faussant ainsi le message du film original et créant des problèmes de communication interculturelle. Susanne Niemeier explique, à propos de la version doublée du film en allemand:

If a German spectator does not know the relevance of a sign which is based on American traditions [the tension between the North and the South represented by the conflict between Stanley and Blanche, for example], this sign will be considered as a sign per se, i.e. as a mere sensual impression, but it will escape the interpretation intended by the author/director due to its lack of denotatum for people of different cultures. (1991: 147)

\subsection{Le contexte géographique}

Le contexte géographique est l'élément le plus chargé culturellement du film, d'autant plus que la ville de la Nouvelle-Orléans est depuis longtemps déphasée par rapport au reste des États Unis, aussi bien sur le plan culturel que géographique. Le nom des tramways, "Desire» et "Cemeteries», et celui de la rue "Elysian Fields», qui apparaissent au début du film, existaient déjà du temps de Tennessee Williams et participent à l'évocation du décor particulier de la ville. Les deux noms «Desire» et «Cemeteries» ont été traduits littéralement dans les deux versions françaises («Désir» et «Cimetières»). Bien que cela pose un problème de cohérence référentielle (referential accuracy), il faut souligner que les deux noms symbolisent les deux leitmotivs de l'histoire - le désir et la mort - et qu'il était nécessaire que cela soit marqué de manière évidente aux yeux du spectateur francophone. La traduction de «Elysian Fields» est toute aussi intéressante puisque le nom a lui-même un référent précis dans la culture française. Le sous-titreur a choisi de garder la version anglaise (qui a le mérite de conserver la cohérence référentielle mais pose un problème de cohérence interne puisque les deux autres noms propres ont été traduits), alors que le doubleur a choisi "Champs-Élysées». Dans la scène où Blanche rencontre Eunice au début du film, celle-ci lui dit en effet: "Mais vous y êtes aux Champs-Élysées!», ce qui, même si l'allusion ironique au fameux boulevard parisien a ici le mérite d'être conservée, pose un problème de référent culturel. La réplique d'Eunice ne peut que frapper le spectateur français qui associe inévitablement d'autres images à ce nom des images totalement étrangères au décor si particulier à la Nouvelle-Orléans que Williams et Kazan ont voulu présenter.

Plusieurs autres références à des endroits réels de la Nouvelle-Orléans sont faites plus tard dans le film. La version doublée semble avoir essayé d'éviter toute référence à l'anglais, ce qui a pour effet de naturaliser les réalités culturelles: «Bourbon Street», par exemple, la célèbre avenue associée au quartier français, devient «le Boulevard Bourbon» (alors que les sous-titres ont conservé «Bourbon Street»). Le nom 
«Galatoire's », l'un des plus vieux et plus célèbres restaurants de la Nouvelle-Orléans, disparaît des deux versions françaises, où Blanche et Stella vont seulement au restaurant. Nous pouvons cependant considérer que, dans ce cas, le référent culturel n'était pas assez connu du public français pour mériter d'être conservé. Si l'on analyse les choix du sous-titreur dans leur ensemble, c'est-à-dire le fait qu'il ait conservé l'anglais «Bourbon Street», laissé de côté la référence à «Galatoire’s» et traduit « to the French market» par au marché, on réalise finalement que l'ambiance française de la Nouvelle-Orléans n'est plus aussi apparente qu'elle ne l'était dans la version originale du film. Or Williams voulait justement essayer de suggérer «[the] raffish charm [of] this once noble and now quite delapidated quarter» (Niemeier, 1991: 154). Laisser de côté ces références ou manquer de cohérence dans leur traduction peut, par conséquent, jouer un rôle sur la représentation que les spectateurs se font du cadre géographique au début du film.

\subsection{Le contexte historique}

En ce qui concerne le contexte historique, le conflit symbolique entre le Nord et le Sud des États-Unis, manifesté par la confrontation entre Stanley et Blanche, joue aussi un rôle majeur dans la signification du film, puisque Tennessee Williams voulait symboliser l'anéantissement du vieux Sud décadent par la nouvelle société industrialisée du Nord. Cette allusion voilée à la guerre civile est assez évidente aux yeux d'un spectateur américain, mais l'est beaucoup moins aux yeux d'un public étranger, posant ainsi un problème aux traducteurs. Au début du film, lorsque Blanche et Stella parlent de Stanley, Stella suggère en effet: "Of course there were things to adjust myself to later on... » et Blanche réplique immédiatement: "Such as his civilian background...». Cette allusion aux origines et aux valeurs différentes de Stanley devient: «Comme le milieu où il vit...» dans les sous-titres, et disparaît de la version doublée pour être remplacée par «Oui, je m'en doute!...», ce qui apparaît beaucoup plus ironique de la part de Blanche et fait totalement disparaitre l'allusion sociohistorique. Ainsi, lorsque Blanche s'adresse à Stanley et s'exclame, dans la version doublée: «...dans vos pattes de paysan! » pour traduire «...in your capable hands», le choix est particulièrement malheureux puisque Stanley est supposé représenter, au contraire, le monde urbain et industriel du Nord en train d'écraser la société des planteurs du Sud. Cette traduction, bien qu'elle puisse probablement s'expliquer par la contrainte du synchronisme (lip synchronization) (en particulier pour reprendre les sons /p/ et /b/ de la version originale anglaise, deux bilabiales très évidentes au mouvement des lèvres) ne respecte cependant pas le symbolisme voulu par Tennessee Williams.

\subsection{Le contexte social}

Pour évoquer le contexte sociohistorique particulier dans lequel se déroule l'histoire, Williams utilise un contraste d'accents et de registres de langue. La question des accents, en tant qu'éléments spécifiques à une culture ou un groupe, pose des difficultés à tout traducteur. Le contraste d'accent entre Marlon Brando (qui parle avec un accent new-yorkais) et Vivien Leigh (qui s'exprime avec l'accent traînant du Sud) est inévitablement perdu dans la traduction française ${ }^{6}$. Cette opposition si fondamentale au symbolisme du film a cependant été transposée avec succès sur le plan du 
registre de la langue. Pour tenter de suggérer le contraste entre la façon de parler de Stanley et celle de Blanche, le sous-titreur et le doubleur ont tous deux essayé de rendre, d'une part, la familiarité et la vulgarité du langage utilisé par Stanley et, d'autre part, le registre de langue soutenu et parfois lyrique employé par Blanche. Lors de la première rencontre entre les deux personnages, Stanley s'exclame: "Ah oui, la sœur à Stella!» et «J'ai ma ch'mise qui m'colle après!» dans la version doublée, et nous trouvons des mots comme «Salut!», «la gnôle» et «crécher» dans les sous-titres. Des signes du langage parlé apparaissent aussi dans les sous-titres, comme par exemple: "C'est pas...» (omission de «ne»). Ces choix montrent les efforts faits par les traducteurs pour trouver des expressions familières destinées à dénoter les origines ouvrières de Stanley. Blanche, quant à elle, utilise, comme dans la version originale, un registre de langue élevé suggérant ses origines aristocratiques: «Faites, je vous en prie» dans la version doublée, «faire bonne figure» et "vous me voyez ainsi» dans les sous-titres. Malgré quelques décalages de registre (par exemple, lorsque Blanche demande à sa sœur: "C'est un chaud lapin?» (à propos de Mitch) pour traduire: "Is he a wolf?», ce qui semble surprenant de la part de Blanche), sa façon de parler a dans l'ensemble été respectée.

Il semble donc que la perte des accents ait été compensée par un contraste du registre de la langue, ce qui, selon moi, est une meilleure solution que celle proposée par Neimeier, pour la version doublée en allemand: "Stanley could have spoken with a German working-class accent, possibly of the Ruhr area, which is generally associated with working-class people, and Blanche could have spoken 'high' German with a posh accent, e.g. from Hamburg» (1991: 155). Cette solution reviendrait en effet à naturaliser de façon artificielle une réalité culturelle spécifiquement étrangère et pose surtout un problème de cohérence langue/culture. C'est ce que Hans Vöge expliquait déjà clairement en 1977: "The subject of a film is a specific situation intimately connected with a specific place and a specific time. The factor of place and time together determine what language is spoken in the specific situation» (1977: 123).

\subsection{Références au français}

Enfin, il peut être intéressant d'analyser ce que les références à l'héritage français, dont le film abonde, sont devenues dans les traductions. Comme ces références à la langue française permettent de nouveau d'évoquer l'ambiance particulière de la Nouvelle-Orléans, il semble important de les garder. Là encore, le public américain comprend immédiatement la portée de ces allusions, ce qui n'est pas forcément le cas des spectateurs étrangers. Le nom de la plantation Belle Reve conserve ses origines françaises dans les sous-titres, où l'accent a été réintroduit (Belle Rêve), mais, dans la version doublée, de façon surprenante, le nom est prononcé /bel ri:v/, c'est-à-dire avec un accent anglophone ${ }^{7}$, de sorte que la référence au monde de rêves et d'illusions de Blanche disparait, d'autant plus que le mot «rive» renvoie à des images totalement différentes. Un autre passage intéressant est lorsque Blanche DuBois explique la signification de son nom à Mitch: "It's a French name. It means 'woods', and Blanche means 'white'. So the two together mean 'white woods'. Like an orchard in Spring." Évidemment, toute traduction de ce passage en français est inévitablement un peu maladroite puisque l'explication ne se justifie plus. Les versions sous-titrée et doublée ont tout de même décidé de ne pas la supprimer. La version sous-titrée propose: 
"C'est un nom français, qui parle de forêts. Et mon prénom est Blanche. Je suis la Blanche Forêt, tel un verger au printemps", alors que la version doublée énonce: «C'est un nom français. Comme la forêt... et blanche comme la dame [?]. Les deux ensemble signifient 'Forêt Blanche', les arbres au printemps », ce qui manque de toute évidence de cohérence.

En conclusion, le sous-titrage et le doublage ont tous deux dû faire face à des problèmes de transferts culturels. Le doubleur a décidé de naturaliser la plupart des allusions à la réalité sociohistorique américaine, d'où les problèmes de cohérence entre les choix lexicaux et les signes visuels du film. Ceux-ci appartenant au monde culturel des spectateurs d'origine (américains, dans ce cas) ne correspondent plus aux signes linguistiques (français, ici) utilisés pour les décrire. Le sous-titreur, lui, s'est appuyé davantage sur l'interdépendance entre les signes verbaux et le contexte énonciatif plus large, conservant ainsi davantage le caractère étranger de l'original. Or, si dans certains films le contexte socioculturel d'origine ne joue qu'un rôle secondaire, il est ici un élément fondamental de l'histoire et il est alors important de garder les allusions qui y sont faites. Comme les sous-titres permettent de mieux préserver l'authenticité du rapport langue/culture, ils semblent donc mieux fonctionner pour ce type de film. Malheureusement, la lecture des critiques de A Streetcar Named Desire publiées lors de sa sortie en France ne permet pas de confirmer cette hypothèse.

\section{Présentation des personnages et de leurs relations}

En plus de cet ancrage sociohistorique très fort, le film repose également en grande partie sur les interactions entre les personnages (d'autant plus qu'il ne faut pas oublier que le film a pour origine une pièce de théâtre). Comme pour la question des transferts culturels étudiée précédemment, la façon dont la version sous-titrée et la version doublée présentent les personnages et leurs relations peut avoir des conséquences non négligeables sur l'image que s'en font les spectateurs et, par conséquent, la réception du film en dépend. Le décor, en effet, varie peu - la plupart de l'action se déroulant dans l'appartement des Kowalski; l'attention est plutôt portée sur le jeu des personnages et en particulier sur la confrontation, principalement verbale, entre Stanley et Blanche.

\subsection{Blanche B $^{8}$}

L'analyse contrastive des trois versions montre que certains changements dans les deux versions françaises, apparemment mineurs, peuvent modifier l'impression générale laissée par Blanche. Elle ne semble, en effet, pas aussi hésitante que dans l'original: dans les sous-titres, ceci est dû principalement au fait que les marqueurs d'hésitation (habituels à Blanche) sont systématiquement perdus lorsqu'ils sont transférés dans le mode écrit (Mason [1989], Hatim \& Mason [1997], Kovacic [1998]). Or, comme le fait remarquer Kovacic: "Similarly indicative of a person's state of mind are hesitations, false starts, incomplete sentences and other similar features of spoken language» (1998: 81). Ces éléments suggèrent la fébrilité de Blanche, dès le début du film. Lorsque, par exemple, Stella et Blanche se retrouvent au bowling et que Stella veut présenter Stanley à sa sœur, les hésitations de Blanche: "Oh no, not now, not now... » deviennent dans les sous-titres: "Pas tout de suite», et «Which is 
he, which one is he... ?» est rendu par: «Lequel est-ce?». Un spectateur qui s'appuierait principalement sur les sous-titres ne pourrait pas deviner l'embarras et les hésitations de Blanche à ce moment-là, si ce n'est grâce au jeu de l'actrice. Comme le laissent entendre Lambert et Delabastita, «le véritable discours [a] lieu ailleurs, c'està-dire sur l'écran» (1996: 54). C'est peut-être pour cette raison que le sous-titreur a décidé de ne pas représenter les répétitions par écrit.

De plus, quelques choix de traduction donnent l'impression que Blanche est moins fragile: lors de son premier tête-à-tête avec Mitch, au cours duquel les deux personnages discutent des gens qui souffrent et des sentiments qu'ils peuvent éprouver («The little there is belongs to people who have experienced such sorrow» / «I believe you're right about that»), le doubleur fait de cette conversation d'ordre général une discussion plus personnelle: "On dirait que vous avez souffert», dit Mitch à Blanche, qui répond: "L'ai un peu d'expérience!». Blanche n’apparaît donc pas aussi évasive que dans la version originale. Plus tard, elle explique à Stella: "Je f'rais tout pour ne pas le décevoir... afin qu'il [Mitch] m’épouse », alors que, dans la version anglaise, l'on trouve: "I want to deceive him just enough to make him want me». En plus du faux-sens dans la première partie de la phrase, la conjonction 'afin que' donne l'impression que Blanche a une idée précise en tête et qu'elle fera tout pour arriver à son but. Vis-à-vis de Stanley, Blanche apparaît également un peu plus ironique et plus sûre d'elle. La remarque: "You, healthy Polack, certainly don't... », par exemple, devient une interjection: «Espèce de Polack! Espèce de sauvage!». Elle qualifie également Stanley de «buté » et «à l'emporte-pièce» au lieu de «a little bit on the primitive side, I should think». Pour cette réplique, les sous-titres offrent un intéressant point de comparaison puisque, comme l'on pouvait s'y attendre, l'expression d'atténuation (down-toner $)^{9}$, "I should think», disparait, ce qui rend la remarque beaucoup plus directe: "avec des tendances un peu primaires». Blanche apparait ainsi comme un personnage plus déterminé, moins mystérieux, subtil ou fragile que dans la version originale.

De plus, son côté nymphomane est légèrement renforcé dans la version doublée: Stanley «est un amour de s'être occupé de [s] es bagages » (alors que le synchronisme ne peut pas être en cause dans cette phrase, puisque Blanche est cachée dans la salle de bain) au lieu de simplement: «It was so good of Stanley to call for my trunk». De plus, Blanche, en réponse à une remarque de Mitch, ne s'exclame pas: "J'apprécie votre galanterie » comme dans les sous-titres, mais: «Je suis très sensible à la galanterie!», ce qui est une remarque d'ordre général sur ses relations avec les hommes. En insistant sur le côté nymphomane de Blanche, le doubleur n'ajoute donc pas un aspect nouveau à la personnalité de Blanche puisque ce côté va être confirmé plus tard dans le film, mais il rend le personnage moins évasif et moins complexe.

Enfin, Blanche apparaît également plus sûre d'elle envers sa sœur, Stella ${ }^{10}$. "I've been half crazy!» devient, dans la version doublée: "Je n'ai pas dormi de la nuit à cause de toi ». Au tout début du film, l'hésitation "It's not what...» devient «Ne te fâche pas, ma chérie! », beaucoup plus direct, et "Take into consideration... you left» est doublé par: «Tu n'as songé qu'à toi!», qui est de nouveau plus accusateur. Dans la version sous-titrée, c'est l'omission répétée (pour des raisons de contraintes de temps et d'espace) de termes d'affection entre les deux sœurs, servant habituellement de down-toners lorsque la conversation devient trop dure, qui donne cette impression que Blanche est plus agressive envers Stella. En effet, comme le souligne Mason: 
"Modality and affective meaning are conveyed by the use of all kinds of short expressions which serve to reinforce or attenuate the force of what is being said» (1989:21). Par exemple, lorsque Blanche accuse sa sœur de n'être revenue à Belle Reve que lors des enterrements, les expressions «Stella, my sister» ou «honey» disparaissent complètement des sous-titres.

\subsection{Stanley}

En ce qui concerne Stanley, sa personnalité n'est pas non plus transformée radicalement par le processus de traduction. Mais la version doublée a tendance à exagérer sa vulgarité: à plusieurs occasions, Stanley utilise des mots d'un registre plus grossier que dans l'original. Ainsi, lors de sa première apparition c'est-à-dire lorsque le spectateur commence à se former une image du personnage, au lieu de traduire plus ou moins littéralement: "Hey, Stella, what did you do, fall asleep in there?», comme le sous-titreur a choisi de le faire («Tu dors là-dedans ou quoi?»), le doubleur fait dire: «Eh, Stella, qu'est-ce que tu fous, bon dieu? T'es tombée d'dans ou quoi ?», alors que Stanley n'est pas visible à l'écran à ce moment-là - la contrainte de la synchronisation ne peut donc être évoquée ici. Plus loin, «Hush up!» est doublé par «Fermez vos gueules» (en voice-over), «Hi ya Stella!» par «Ça gaze oui?» (plus désinvolte) et, dans la scène du viol de Blanche, «Tiger! Tiger!» (rappelant symboliquement à la fois les chats errants qu'on entendait au début du film, l'image du personnage de Stanley qui est tel «un fauve, à l'étroit dans le cadre de l'écran» (Vidal, 1987) et l'une des significations argotiques du mot 'cat' : celle de 'prostituée' [Zuber,1980:68]) est traduit par «Quelle chatte! Quelle chatte! Salope!», transformant ainsi ce qui était une allusion symbolique en une insulte vulgaire.

Dans ses relations avec les deux femmes, Stanley subit également une légère transformation dans les versions traduites. Le personnage apparaît plus ironique envers Blanche dans la version doublée, la surnommant «la pauvre mignonne» ou «la duchesse », expressions ironiques absentes de l'original. Puis, au lieu de répondre à Stella qui lui demande: «Don't I rate a kiss?», "Not in front of your sister!», Stanley, dans la version doublée, dit d'un ton ironique: «Non, je ne peux pas faire ça devant madame! ». Ajouté au fait que Stanley ait l'air plus vulgaire et plus ironique, il semble également qu'il apparaisse plus agressif dans la version doublée, comparé à la version originale. Ceci est dû au fait que les tensions entre Stanley et Stella sont accentuées. Le traitement de la phrase cruciale: "The Kowalskis and the DuBois just have got a different notion on this» est particulièrement intéressant sur ce point. Les deux versions françaises ont décidé de transformer cette affirmation spécifique («on this») en une affirmation d'ordre général: «On n’a pas la même conception des choses!» (version sous-titrée) et «Faut croire que les Kowalski et les DuBois ont des points de vue qui ne s'accordent pas!» (version doublée), ce qui suggère que les tensions dans le couple datent probablement d'un certain temps, alors que T. Williams voulait laisser entendre au contraire que les tensions entre Stella et Stanley n'avaient réellement commencé qu'après l'arrivée de Blanche. En somme, de tels détails sont susceptibles de donner aux spectateurs français une impression légèrement différente des interactions entre ces protagonistes du film. 


\subsection{Mitch}

Finalement, le personnage de Mitch subit aussi quelques transformations dans la version doublée par rapport à la version originale. En plus de la question de l'oralisation sur laquelle nous aurions pu nous attarder (en particulier le ton employé par l'acteur choisi pour doubler Karl Malden), ce sont principalement des expressions telles que «le bid», «jusqu'à ce que je sois casé » et «C’est ce que je pèse à poil » qui semblent mal à propos dans la bouche de Mitch. Certains choix linguistiques le rendent également moins timide et moins courtois que dans l'original. L'échange entre Mitch et Blanche: «How do you do?» / «How do you do, miss DuBois?», lors de leur première rencontre, est rendu dans la version doublée par: «Ravi de vous connaître» / "Comment ça va, mademoiselle? », qui ne suggère pas l'échange très formel et presque guindé de la version originale. Dans les sous-titres, cet échange n'est même pas traduit, ce qui confirme les conclusions de Hatim et Mason au sujet des formules de politesse, mais rappelle que de telles décisions peuvent avoir des conséquences sur la perception des interactions entre les personnages. Dans cette scène, cela est d'autant plus important que la formule utilisée par Mitch permet de le distinguer des autres hommes qui n’ont pas salué Blanche. Nous pouvons remarquer, comme Mason, que:

Cumulatively, the absence of the politeness features which we have discussed cannot fail to convey a different idea of the personality of the characters on screen and of their attitudes towards each other. Items which, taken in isolation, appear to be of relatively little significance turn out to be powerful indicators of rhetorical purpose and the dynamics of interaction (1989: 24).

Dans la scène suivante, les excuses répétées de Mitch auprès de Blanche, «Excuse $m e »$ (deux fois), «Sure», etc... ne sont pas non plus traduites, alors qu'elles évoquent l'embarras des personnages et la formalité de leurs relations. Comme Mason et Hatim l'ont conclu pour Un cour en hiver, "It is difficult for the target language auditors to retrieve interpersonal meaning in its entirety. In some cases, they may even derive misleading impressions of characters' directness or indirectness » (1997: 444).

\section{En guise de conclusion}

Mon but n'a pas été de critiquer l'un ou l'autre des modes de traduction audiovisuelle, mais d'analyser "the kinds of meanings which tend to be omitted and [...] the effects such omissions may have» (Hatim \& Mason, 1997: 444) ${ }^{11}$. Dans les deux modes, le contenu propositionnel (propositional meaning) a été traduit, mais la non-traduction ou la modification de certains éléments appartenant à la fonction phatique ou émotive du langage peut entraîner, par effet cumulatif, une perception différente du film.

La question des transferts culturels semble avoir été principalement un problème pour le doubleur, qui a choisi - probablement parce que le doublage, en raison de sa nature, a déjà tendance à effacer le caractère étranger du décor - à naturaliser davantage les références culturelles que le sous-titreur. Dans le cas d'un film comme A Streetcar Named Desire, où presque chaque allusion culturelle a une justification symbolique ou esthétique, naturaliser les référents revient à modifier tout un réseau symbolique de signes verbaux et non verbaux. 
En ce qui concerne la présentation des personnages, il est difficile de dire si l'un des modes de traduction audiovisuelle a plus d'effets divergents que l'autre. La version doublée, par certains choix linguistiques, en particulier des décalages de registre de langue et de ton comparé aux complexités des personnages dans la version originale, semble avoir nivelé les personnages, faisant par exemple de Blanche une femme plus sûre d'elle et moins fragile et de Stanley un personnage plus agressif et plus grossier. Or ces changements ne peuvent pas tous s'expliquer par l'argument du synchronisme, souvent avancé. Le sous-titrage, lui, n’a pas eu les mêmes conséquences sur la présentation des personnages. Le fait que les éléments dénotant les relations interpersonnelles (en particulier les termes d'affection, les formules de politesse et les marqueurs d'hésitation) sont souvent les premiers à être éliminés dans les sous-titres, a par accumulation un impact sur la façon dont certains personnages peuvent être perçus par le spectateur. Les conclusions de Hatim et Mason semblent donc confirmées dans ce film, mais, comme pour Un cour en hiver, cela est dû, à mon avis, au fait que ces marqueurs linguistiques sont particulièrement significatifs pour ces deux films, dans lesquels les interactions entre les personnages sont plus importantes que l'action per se (ce qui n'est pas bien sûr le cas de tous les films). Comme le fait remarquer Kovacic:

When films, dramas or comedies, in which personal and interpersonal components are fundamental dramatic devices, are subtitled according to the priorities of written discourse (with the disappearance of characteristics of spontaneous speech [...]), the shift may have significant consequences for the comprehension of the dramatic or comic development of the story, the relationships between characters, and their psychological states (1996: 107).

Il est donc important, pour chaque film, que le traducteur-adaptateur en identifie tout d'abord les fondements esthétiques et symboliques et, si besoin est, qu'il privilégie parfois la traduction d'éléments de pragmatique interpersonnelle sur la traduction du contenu propositionnel.

En somme, si le sous-titrage a entraîné principalement des pertes, le doublage a provoqué surtout des changements (certains radicaux). La version sous-titrée apparaît finalement plus proche de l'original, probablement parce que le rythme relativement lent des dialogues et le fait qu'il y ait assez peu d'action susceptible de détourner l'attention du spectateur permettent au lecteur, la plupart du temps, de suivre facilement les sous-titres. Dans l'ensemble, la version sous-titrée semble mieux adaptée à ce type de film, où les questions de cohérence langue/culture, de registre de langue, de complexité des personnages et de relations interpersonnelles sont cruciales pour la signification de l'histoire. N'oublions pas, cependant, que le sens au cinéma est le résultat d'éléments verbaux aussi bien que visuels et sonores. La composante verbale n'est donc qu'un élément parmi d'autres. Or, comme le souligne Irena Kovacic: "Very little is known at the present stage about the interaction of subtitles and picture in the viewer's reception of television programmes» (1998: 81), ceci s'appliquant aussi au doublage. Le fait que les critiques françaises du film que j'ai lues ne fassent en aucun cas référence à la traduction suggère soit que les divergences soulignées entre la version originale et les versions traduites ne jouent pas un rôle majeur dans la perception du film, soit que le non-verbal (en particulier le jeu des acteurs, le décor et la musique) relaie suffisamment l'élément verbal. C'est ce que semble suggérer cet extrait d'une des critiques du film: «Brando n'avait pas besoin de parler pour exprimer des sentiments 
complexes autant que violents» (Guez: 1987). Il reste donc à approfondir les interactions entre image et langue, en particulier à mieux appréhender leur effet sur la réception d'un document audiovisuel.

\section{NOTES}

1. Le DVD utilisé pour cette étude est celui sorti en 1997 (Warner Studios).

2. Je dois souligner ici qu'il m'a été impossible de trouver, malgré mes recherches, les références exactes (date de réalisation et nom des traducteurs) des versions avec lesquelles j'ai travaillé. Ceci démontre, si besoin est, le peu de reconnaissance encore accordé à la traduction audiovisuelle.

3. Les deux auteurs avaient conclu que, à cause des contraintes techniques imposées au sous-titrage, les éléments interpersonnels (dans le cas de leur étude, principalement les formules de politesse et les marqueurs de modalité) tendent à disparaître dans les sous-titres, influençant ainsi l'image que les spectateurs se font des relations entre les personnages.

4. Voir sur ce point l'article de Martine Danan (1999).

5. Je reprends certaines de ces catégories de Niemeier (1991).

6. Jean Cocteau, dans son adaptation libre de la pièce en 1949, avait choisi de faire parler Stanley avec un argot parisien, mais il s'agit d'un exemple de choix radicaux et assez rares (Kolin: 2000).

7. Ceci est peut-être dû au fait qu'un adjectif au féminin avec un nom au masculin aurait été maladroit en français.

8. Je me concentrerai sur les personnages principaux.

9. Je reprends ce terme de Mason (1989).

10. Les changements apportés au personnages de Stella dans les versions traduites ne me paraissent pertinents que dans le cadre de ses relations avec Blanche.

11. Notons au passage que beaucoup d'autres éléments auraient pu être étudiés dans cette même perspective constrastive. Comme par exemple les questions d'oralisation (choix de la voix pour doubler et ton employé), la cohérence entre traduction et images (gestes, mimiques des personnages), l'ordre des mots et les marques d'oralité dans les sous-titres, la question de la traduction des noms propres, etc.

\section{RÉFÉRENCES}

Danan, M. (1999): "Subtitling: Multiculturalism or Commodification of Culture?", Comparative Literature Now: Theories and Practice, S.T. de Zepetnek, M.V. Dimic and I. Sywenky (eds.), Paris, Champion, pp. 763-773.

Delabastita, D. (1990): "Translation and the Mass Media", Translation, History and Culture, S. Bassnett \& A. Lefevere (eds.), London, Pinter, pp. 97-109.

Guez, G. (1987) : «Brando: naissance d'un mythe», Le Figaro, numéro du 13 juin.

Hatim, B. and I. Mason (1997): "Politeness in Screen Translation", The Translation Studies Reader, L. Venuti (ed.) (2000), London \& New York, Routledge, pp. 430-45.

Kolin, P. (2000): Williams - A Streetcar Named Desire: Plays in Production, Cambridge, Cambridge University Press.

Kovacic, I. (1996): "Reinforcing or Changing Norms in Subtitling", Teaching Translation and Interpreting 3, C. Dollerup and V. Appel (eds.), Amsterdam, Benjamins, pp. 105-9.

Kovacic, I. (1998): "Six Subtitlers - Six Subtitling Texts", Unity in Diversity? Current Trends in Translation Studies. L. Bowker, M. Cronin, D. Kenny and J. Pearson (eds.), Manchester, St Jerome, pp. 75-82.

Lambert, J. et D. Delabastita (1996): «La traduction de textes audiovisuels: modes et enjeu culturel», Les transferts linguistiques dans les médias audiovisuels, Yves Gambier (ed.), Villeneuve d'Ascq, Presses universitaires du Septentrion, pp. 33-58.

LANG, G. (1996): «Prolégomènes à l'étude du doublage», Language and Literature Today (Proceedings of the XIXth Triennial Congress of the International Federation for Modern Languages and Literatures), Brasilia, Universidade de Brasilia, pp. 398-402.

Mason, I. (1989): "Speaker Meaning and Reader Meaning: Preserving Coherence in Screen Translating", Babel: The Cultural and Linguistic Barriers Between Nations, R. Kölmer \& J. Payne (eds.), Aberdeen, Aberdeen University Press, pp. 13-24. 
Niemeier, S. (1991): "Intercultural Dimensions of Pragmatics in Film Synchronisation", The Pragmatics of International and Intercultural Communication, J. Blommuert \& J. Verschueren (eds.), Amsterdam, Benjamins, pp. 145-162.

Vidal, M. (1987): «Apporte-moi des orages», Télérama, numéro du 13 juin.

Vöge, H. (1977): "The Translation of Films: Sub-Titling versus Dubbing”, Babel, 23-3, pp. 120- 125.

Zuber, O. (1980): “The Translation of Non-Verbal Signs in Drama”, Pacific Quarterly Moana, 5-1, pp. $61-74$. 